\title{
PREVENTION
}

\section{Relating risk factor reduction to disease risk on a global level}

In 2013, the WHO member states adopted a $25 \%$ mortality reduction target for four main noncommunicable diseases (NCDs, defined as cardiovascular disease, chronic respiratory disease, cancers, and diabetes mellitus) by the year 2025. Included in this target were reductions in key risk factors for these diseases (tobacco and alcohol use, salt intake, obesity, blood pressure, and blood glucose). However, "It was not known if achieving the risk factor targets would lead to achieving a $25 \%$ NCD reduction by 2025 ” points out Robert Beaglehole, a senior author of the first integrated analysis of how WHO NCD reduction targets relate to each risk factor. The report was published online in The Lancet in May 2014.

If achieved, 25\% NCD reduction would theoretically delay or prevent $>37$ million deaths by 2025 . This study suggests that meeting risk factor reduction targets alone would, in reality, lead to only a $22 \%$ and $19 \%$ decrease in NCD mortality in men and women, respectively, compared with $11 \%$ and $10 \%$ in a "business as usual" scenario. Tobacco use proved to be the most conspicuous NCD risk factor, "if countries adopt a more ambitious tobacco reduction target (50\% not $30 \%$ ), we can achieve the mortality target in men and get very close in women" says Beaglehole.

Results also show that low-to-middle income countries achieving these targets would prevent or delay 31 million deaths by 2025 . The authors concluded that WHO targets should be taken seriously by all countries, but further actions are also needed. Rapid interventions should include tobacco control, and early detection and treatment programmes.

Peter Sidaway

Original article Kontis, $V$. et al. Contribution of six risk factors to achieving the $25 \times 25$ non-communicable disease mortality reduction target: a modelling study. Lancet doi:10.1016/S0140-6736(14)60616-4 